\title{
Evidencia de validez de constructo para el WISC-IV con muestra brasileña*
}

\section{Construct Validity of the WISC-IV among a Brazilian Sample}

Recepción: 29 Noviembre 2013 | Aprobación: 15 Julio 2016

\author{
Fabián Javier Marín Rueda** \\ Universidade São Francisco, Brasil \\ Acácia Aparecida Angeli dos Santos*** \\ Universidade São Francisco, Brasil \\ Ana Paula Porto Noronha**** \\ Universidade São Francisco, Brasil
}

**Doctor en Psicología por la Universidade São Francisco, Itatiba/SP. Docente del Programa de Pos-Grado en Psicología de la Universidad São Francisco. Becario del CNPq-Brasil.

***Doctora en Psicología por la Universidade de São Paulo. Docente del Programa de Pos-Grado en Psicología de la Universidad São Francisco. Becaria del CNPq-Brasil.

****Doctora en Psicología por la Pontifícia Universidade Católica de Campinas/SP. Docente del Programa de Pos-Grado en Psicología de la Universidad São Francisco. Becaria ldel CNPqBrasil.

\section{RESUMEN}

El objetivo fue analizar la estructura interna de la versión brasileña del WISC-IV, comparando el modelo de puntuaciones compuestas con otros dos modelos, el de un factor general de la inteligencia y un modelo con base en la teoría CHC de Cattell-Horn-Carroll. Participaron 795 niños y adolescentes de diferentes regiones de Brasil, con edades entre 6 y 16 años. Los resultados mostraron que el modelo que mejor se ajustó fue el propuesto por el manual del WISC-IV. El modelo con base en la teoría CHC también presentó buen ajuste, pero con valores un poco inferiores a la propuesta original. El modelo de un factor general fue rechazado, pues los índices de bondad de ajuste no fueron satisfactorios. Se sugieren futuras investigaciones que estudien la estructura del WISC-IV en otros países.

Palabras clave

inteligencia, escalas Wechsler, validez, estructura interna.

\begin{abstract}
This study analyzed the internal structure of the Brazilian version of the WISC-IV by comparing the composed scoring model with two other models: one based on a general factor of intelligence and another based on the CHC theory. The participants included 795 children and adolescents between the ages of 6 and 16 hailing from different regions of Brazil. The results indicated that the most suitable model was the one proposed in the manual. The model of the CHC theory also presented a good fit, but with values that was inferior to those of the original proposal. Moreover, the model of the general factor was rejected because the goodness-of-fit indices were not satisfactory.
\end{abstract}

Keywords

intelligence, Wechsler scales, validity, internal structure.

Para citar este artículo: Marín Rueda, F. J., Santos, A. A. A., \& Noronha, A. P. P. (2016). Evidencia de validez de constructo para el WISC-IV con muestra brasileña. Universitas Psychologica, 15 (4). http://dx.doi.org/10.11144 /Javeriana.upsy15-4.evem 
Fabián Javier Marín Rueda, Acácia Aparecida Angeli dos Santos, Ana Paula Porto Noronha.

En el comienzo del siglo XX Charles Spearman $(1904,1927)$ elaboró una teoría de la inteligencia y el método de análisis factorial para comprobarla, siendo sus postulados aceptados actualmente. Él creía que un gran dominio del conocimiento podría ser explicado por un pequeño número de variables. Spearman (1904) consideraba que la inteligencia estaba formada por un factor general $(\mathrm{g})$ y por factores específicos (s). De acuerdo con el autor, el factor $\mathrm{g}$ se caracterizaría por ser constante e inalterado en cada individuo, pero podría variar mucho entre un sujeto y otro. A su vez, los factores s podrían variar, no solo entre diferentes sujetos, sino también en el mismo individuo, de acuerdo con la habilidad que estuviese siendo analizada. Posteriormente, en 1927, añadió a su teoría los llamados factores de grupo, que son características que están presentes en más de una habilidad del mismo sujeto. Se originó entonces la llamada Teoría Trifactorial de Spearman. Con su surgimiento, en las décadas siguientes el concepto de la inteligencia ha estado marcado por la discusión sobre la existencia o no de ese factor general que influye en el desempeño de los individuos en términos de dominios cognitivos (Jensen, 1998).

En 1941, Cattell argumentó que la inteligencia estaría compuesta por dos factores, que son la inteligencia fluida (Gf) y la cristalizada (Gc). Años después, Horn (1985) amplió la teoría agregando otros factores, como la memoria de corto plazo (Gsm), la inteligencia/procesamiento visual (Gv), el almacenamiento y recuperación de largo plazo (Glr), entre otros. La revisión de Carroll (1993) sobre la inteligencia incluyó la comparación de los resultados de diferentes análisis factoriales realizados a lo largo de décadas de estudio, a partir de lo cual concluyó que la existencia de un factor general era evidente y no podía ser discutida. Este trabajo también permitió fundamentar la afirmación de que la inteligencia tendría una estructura jerárquica compuesta por habilidades específicas agrupadas en diferentes dominios de habilidad cognitiva (Carroll, 1993, 1997).

Las ideas integradas de los tres investigadores dieron origen a la teoría más moderna y difundida sobre inteligencia, el modelo Cattell-Horn-Carroll (modelo CHC) de las habilidades cognitivas (Carroll, 1997). Ese modelo concibe la inteligencia como un constructo multidimensional que abarca diversas capacidades relacionadas a un factor general. Vale destacar que los test de inteligencia surgidos a lo largo de ese período reflejan las concepciones teóricas vigentes, asumiendo frecuentemente la existencia del factor $\mathrm{g}$.

De acuerdo con Schneider y McGrew (2012) el modelo $\mathrm{CHC}$ de las habilidades cognitivas tuvo un gran auge por la consistencia de su concepción de la inteligencia como un constructo multidimensional que abarca diversas capacidades ligadas a un factor general. La teoría CHC también ha sido lo suficientemente sólida como para fundamentar la construcción de otros importantes test de evaluación de la inteligencia, como es el caso del Woodcock-Johnson Test of Cognitive Abilities III (Woodcock, McGrew, \& Schenk, 2007).

También, pruebas tradicionales como la Wechsler Intelligence Scale for Children-fourth edition (WISC-IV) han sido analizadas con base en la perspectiva del modelo $\mathrm{CHC}$, verificando cuánto podría ser explicado del constructo subyacente por medio de los datos ofrecidos por el test (Brenlla, 2013; Keith, Fine, Taub, Reynolds, \& Kranzler, 2006; Watkins, Canivez, James, James, \& Good, 2013). Considerando esto, este trabajo se propone analizar el ajuste de diferentes modelos con base en los resultados de la muestra brasileña de normalización del WISCIV.

Es importante resaltar que la Wechsler Intelligence Scale for Children fue propuesta por Wechsler en 1944, quien entendía la inteligencia como una capacidad global y agregada de un sujeto para actuar con un propósito, pensar de forma racional y relacionarse adecuadamente con su entorno. La escala fue elaborada con el propósito de evaluar los aspectos cognitivos de la inteligencia que Wechsler consideraba más relevantes, a saber: la comprensión verbal, el razonamiento abstracto, el razonamiento perceptivo, el razonamiento cuantitativo y la memoria, y la velocidad de procesamiento. 
Inicialmente, en las primeras versiones del test (WISC, WISC-R, WISC-III), Wechsler (1949, 1974, 1991) presupuso la existencia de dos factores, lo que dio origen a las tareas verbales y de ejecución, y como resultado del desempeño de los niños eran obtenidas tres medidas: el CI verbal, el CI de ejecución y el CI total. En el WISC-IV, la versión más reciente del instrumento, se presenta una estructura que mantiene 10 subtest de la versión anterior, agrega cinco nuevos (Conceptos figurados, Letras y números, Matrices, Animales y Adivinanzas) y elimina otros tres (Completamiento de figuras, Armar objetos y Laberinto). La versión de WISC-IV no incluye las medidas de CI verbal y CI de ejecución, de modo que fueron incorporados conceptos más específicos del funcionamiento cognitivo, que son el Índice de Comprensión Verbal (ICV), el Índice de Velocidad de Procesamiento (IVP), el Índice de Razonamiento Perceptivo (IOP) y el Índice de Memoria Operativa (IMO), además de mantener la evaluación de la inteligencia general, por medio del CI total.

De acuerdo con Brenlla (2013), los cambios ocurridos en el test a lo largo del tiempo pueden ser explicados por la ausencia de una teoría formal que lo sustente, aunque las escalas Wechsler hayan sido construidas aceptando los postulados de Spearman. Con el pasar de los años, el modelo CHC de las habilidades cognitivas ganó una gran aceptación por la consistencia de su concepción de la inteligencia como un constructo multidimensional que abarca diversas capacidades ligadas a un factor general (Schneider \& McGrew, 2012).

El estudio norteamericano de Wechsler (2003) sobre el WISC-IV investigó modelos de uno hasta cinco factores por medio del Análisis Factorial Confirmatorio (AFC), verificando que los modelos con cuatro y cinco factores se ajustaban mejor a los datos. Aunado a lo anterior, el modelo de cuatro dominios fue el más adecuado y respaldado por el instrumento debido a que el modelo de cinco factores no presentó un ajuste mucho mejor al de cuatro ya que uno de los factores fue constituido únicamente por el subtest de aritmética. De esa forma, la estructura del WISC-IV quedó constituida por el Índice de Comprensión Verbal (con los subtest Vocabulario, Comprensión, Semejanzas, Adivinanzas e Información), Índice de Velocidad de procesamiento (subtest Claves, Búsqueda de símbolos y Animales), Índice de Razonamiento Perceptivo (compuesto por los subtest Matrices, Conceptos, Construcción por cubos y Completamiento de figuras) y por el Índice de Memoria Operativa (con los subtest Retención de Dígitos, Letras y números y Aritmética).

Las teorías más actualizadas de la inteligencia, como es el caso de la teoría CHC, confirman que esas habilidades reflejan de hecho aspectos importantes de la cognición (Carroll, 1993, 1997; Horn, 1991). El asunto aquí investigado se refiere a la verificación de la estructura interna del WISC-IV, especialmente el grado en que reproduce el modelo teórico usado como base para su construcción.

Después de la publicación norteamericana del WISC-IV, se han realizado estudios que buscan confirmar su estructura y en comparación con otros modelos alternativos a esta. En ese sentido, serán presentados dos estudios de regiones geográficas diferentes que tuvieron objetivos similares.

En los Estados Unidos fue realizado un estudio por Keith et al. (2006), y en los resultados encontraron que una estructura de cinco factores, fundamentada en el modelo $\mathrm{CHC}$, era más adecuada que la de cuatro dominios, propuesta por el test. De acuerdo con los autores, el modelo de cinco dominios fue el que mostró un mejor ajuste, compuesto por la Inteligencia Cristalizada (subtest Semejanzas, Vocabulario, Comprensión, Información, Adivinanzas y Completamiento de figuras), el Procesamiento Visual (compuesto por Completamiento de figuras, Construcción por cubos, Matrices y Búsqueda de símbolos), la Inteligencia Fluida (con los subtest Matrices, Conceptos y Aritmética), la Memoria a Corto Plazo (Retención de Dígitos y Letras y números) y la Rapidez de Procesamiento Cognitivo (compuesto por los subtest Claves y Animales). Con base en los resultados, los autores sugirieron 
Fabián Javier Marín Rueda, Acácia Aparecida Angeli dos Santos, Ana Paula Porto Noronha.

una reconfiguración de los subtest para la interpretación del WISC-IV, proponiendo que algunos fuesen considerados en más de una dimensión.

Otro estudio sobre el WISC-IV fue realizado con una muestra argentina por Brenlla (2013). La autora tuvo como objetivo verificar la estructura original de cuatro factores propuesta por Wechsler, además de investigar modelos alternativos con base en la teoría CHC, entre los cuales estaba el propuesto por Keith et al. (2006). Por medio del Análisis Factorial Confirmatorio, se logró evidenciar que la estructura de cuatro factores de Wechsler (2003) fue la más adecuada en la muestra argentina, lo cual difiere de los hallazgos de Keith et al. (2006).

Considerando el debate sobre la estructura factorial del WISC-IV en diferentes países del mundo y la reciente adaptación del test en Brasil, el objetivo del presente estudio fue analizar la estructura interna de la versión brasileña del WISC-IV por medio del Análisis Factorial Confirmatorio. Para eso fueron seleccionados tres modelos, a saber: (1) las medidas de puntuación compuestas y cuatro dominios establecidos por el manual del test, (2) el modelo de un factor general de la inteligencia y (3) el modelo $\mathrm{CHC}$, tal como lo propusieron Keith et al. (2006).

\section{Método}

\section{Participantes}

Participaron en la investigación 795 sujetos, de los estados de Minas Gerais, São Paulo y Paraná, en Brasil. Los participantes tenían entre 6 y 16 años de edad $(\mathrm{M}=10.65, \mathrm{DE}=3.06)$ y 53.6 $\%$ era del sexo femenino. La escolaridad estuvo entre el 1..$^{\circ}$ el 9. ${ }^{\circ}$ año de la enseñanza primaria, y las escuelas eran de la red pública de enseñanza. Una descripción más detallada del número de participantes por edad y por sexo se encuentra en la Tabla 1.

\section{Tabla 1}

Frecuencia de participantes por edad y por sexo

\begin{tabular}{crrrr}
\hline Edad & Masculino & Femenino & \multicolumn{2}{c}{ Total } \\
\cline { 4 - 5 } & & & \multicolumn{1}{c}{$F$} & \multicolumn{1}{c}{$\%$} \\
\hline 6 & 35 & 36 & 71 & 8.9 \\
7 & 50 & 44 & 94 & 11.8 \\
8 & 39 & 39 & 78 & 9.8 \\
9 & 31 & 42 & 73 & 9.2 \\
10 & 36 & 40 & 76 & 9.6 \\
11 & 38 & 41 & 79 & 9.9 \\
12 & 38 & 40 & 78 & 9.8 \\
13 & 34 & 34 & 68 & 8.6 \\
14 & 27 & 31 & 58 & 7.3 \\
15 & 26 & 51 & 77 & 9.7 \\
16 & 15 & 28 & 43 & 5.4 \\
\hline Total & 369 & 426 & 795 & 100 \\
\hline
\end{tabular}

Fuente: elaboración propia

La Tabla 1 muestra que hubo una distribución bastante homogénea de la edad de los sujetos, a excepción de la edad de 16 años. También se verifica que hubo una distribución equilibrada de los sujetos por sexo en cada una de las edades.

\section{Instrumento}

La Escala de Inteligencia Wechsler para Niños 4. ${ }^{\circ}$ edición (WISC-IV) está diseñada para evaluar la inteligencia de niños y jóvenes de 6 años a 16 años y 11 meses. El instrumento está compuesto por 15 subtest, a saber: Construcción por cubos, Semejanzas, Retención de Dígitos, Conceptos, Claves A y B, Vocabulario, Secuencia, Matrices, Comprensión, Búsqueda de símbolos A y $\mathrm{B}$, Completamiento de figuras, Animales, Información, Aritmética y Adivinanzas. Para su aplicación, se recomienda que se haga individualmente, en dos sesiones, para evitar el cansancio del examinado (Wechsler, 2003).

$\mathrm{El}$ instrumento ofrece cuatro índices: Comprensión Verbal (CV), Razonamiento Perceptivo (OP), Memoria Operativa (MO) y Velocidad de Procesamiento (VP), además del factor general. El test Comprensión Verbal está compuesto por los subtest Semejanzas, Vocabulario, Comprensión, Información y Adivinanzas; siendo los tres primeros considerados como principales y los dos últimos, suplementarios. OP incluye Construcción por cubos, Conceptos, Matrices y Completamiento 
de figuras, y solo el último es suplementario. Retención de Dígitos, Letras y números y Aritmética conforman el índice MO. Finalmente, el índice VP está compuesto por Código, Búsqueda de símbolos y Animales, siendo el último suplementario.

En lo referente a las propiedades psicométricas, existen diversos estudios de validez y de confiabilidad específicos para la población infantil brasileña. En cuanto a las evidencias de validez, se han realizado investigaciones sobre la estructura interna del test y sobre su relación con otras variables (otra prueba de inteligencia, edad, sexo, evaluaciones docentes, comprensión lectora y reconocimiento de palabras). La confiabilidad fue investigada mediante el método test-retest y mediante el alfa de Cronbach para los diferentes subtest. Tanto los estudios de evidencias de validez como los de confiabilidad presentaron resultados satisfactorios para la muestra brasileña.

\section{Procedimientos}

La recolección de los datos se realizó después de que los padres autorizaron por escrito la participación de sus hijos, así como también fue solicitado el asentimiento de los niños y adolescentes para participar de la investigación. Las escuelas cedieron salas de clase para las aplicaciones que se realizaron a lo largo de dos meses. Para la recolección de los datos, se formó un grupo de siete estudiantes de maestría y doctorado en psicología que pasaron por un entrenamiento riguroso para aplicar la prueba. Cada niño y adolescente que respondió el WISCIV lo hizo en dos días, evitando de esa forma el cansancio. Todas las aplicaciones se llevaron a cabo individualmente, con una duración total de aproximadamente cuatro horas por sujeto, considerando los dos días de aplicación.

\section{Análisis}

Los diferentes modelos se investigaron mediante el Análisis Factorial Confirmatorio (AFC) por medio del programa estadístico AMOS 7, contenido en el SPSS 15 y utilizando el método de máxima verosimilitud. Se estudiaron tres modelos de ajuste: el primero, siguiendo el manual del WISC-IV, de medidas de puntuación compuestas y cuatro dominios específicos y un factor general; el segundo, con un factor general latente producto de los 15 subtests del WISC-IV y el tercero investigando la propuesta de Keith et al. (2006), con base en el modelo CHC.

En el análisis de los modelos y para verificar cuál de ellos se ajustaba mejor, se analizaron siete índices, de acuerdo con recomendaciones señaladas en la literatura (Byrne, 2001; Schweizer, 2010). El primer valor fue el índice de chi-cuadrado (c2), que indica la magnitud de la discrepancia entre la matriz de covariancia observada y la modelada. Considerando que autores como Arbuckle (2009), Arias (1996), Bentler y Bonett (1980) y Tabachnik y Fidell (2001), mencionan que el c2 es afectado por el tamaño de la muestra, no se recomienda emplear solo este índice para evaluar el ajuste del modelo. Por eso, sugieren examinar la magnitud de c2 por medio del índice $\mathrm{c} 2 / \mathrm{gl}$, en el cual los grados de libertad son utilizados para verificar si el valor de c2 es grande o pequeño. De acuerdo con los autores, valores entre 1 y 2 pueden ser considerados excelentes, entre 2 y 3 buenos y solo aceptables valores entre 3 y 5 cuando el análisis se realiza con muestras grandes. Cuando se obtienen valores superiores a 5 se recomienda rechazar el modelo.

También se utilizaron el goodness-of-fit (GFI) $y$ el adjusted goodness-of-fit (AGFI). El primero mide la variancia y covariancia en la matriz observada que está predicha por la matriz reproducida. Son adecuados valores superiores a 0.9. El AGFI indica la proporción de variancia explicada por el modelo, y un valor adecuado también debe ser superior a 0.9. El AGFI es el índice GFI ajustado, considerando los grados de libertad y permite comparar el modelo propuesto con un modelo nulo de referencia, que propone la falta total de asociación entre las variables.

Otro valor considerado fue el comparative fit index (CFI), que calcula el ajuste relativo del modelo al compararlo con el modelo 
denominado nulo, en el cual hay una correlación 0 entre las variables. Un valor superior a 0.9 indica un buen ajuste del modelo. Un concepto análogo al CFI es el NFI, que es un índice de ajuste comparativo y se interpreta como un porcentaje de incremento en el ajuste sobre el modelo nulo, o sea, si el NFI fuera 0.95 , es indicativo de que el modelo propuesto se ajusta $95 \%$ mejor que el modelo nulo. Un valor de referencia para el NFI es que sea superior a 0.9.

El root-square error of aproximation (RMSEA) también fue tomado en cuenta, y se refiere a la raíz de la media de los cuadrados de los errores de aproximación. Valores menores a 0.05 son indicativos de un buen ajuste entre el modelo propuesto y la matriz observada, a pesar de que en muestras grandes sean aceptados valores inferiores a 0.08 .

Por último, se analizó el índice standardized root mean square residual (SMR), que se refiere a la media normalizada de los residuos entre la matriz observada y el modelo. Son indicativos de buen ajuste valores menores a 0.1 .

\section{Resultados}

Considerando el objetivo del estudio, los resultados serán presentados en dos bloques. En primer lugar, las figuras relativas a las soluciones evaluadas para los tres modelos estudiados y posteriormente los datos relativos a los ajustes de esos tres modelos, conjuntamente en una única tabla.

Los datos referentes al modelo propuesto por el WISC-IV, con un modelo jerárquico constituido por un factor general, cuatro factores latentes que se refieren al ICV, IVP, IOP e IMO y 15 variables observadas que se refieren a los subtest, se observan en la Figura 1.

\section{Figura 1}

Solución estandarizada de la estructura factorial de la adaptación brasileña de WISC-IV de acuerdo con el modelo de puntuaciones compuestas.

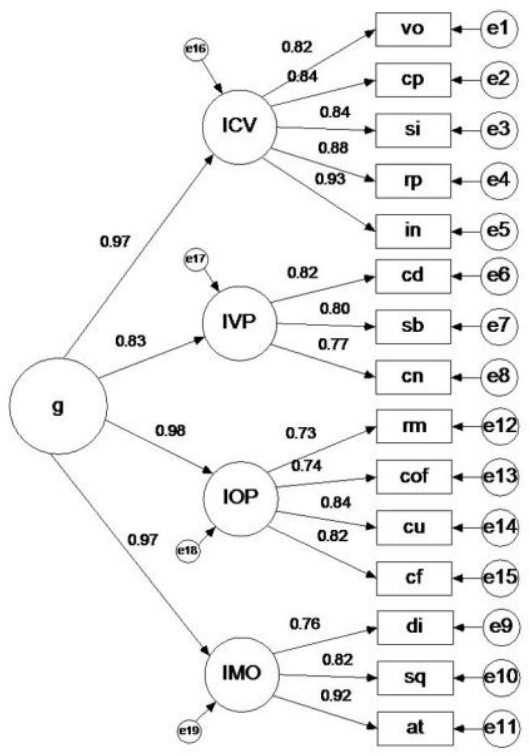

ICV = Índice de Comprensión Verbal / IVP $=$ Índice de Velocidad de procesamiento / IOP = Índice de Razonamiento Perceptivo / $\mathrm{IMO}=$ Índice de Memoria Operativa / $\mathrm{VO}=$ Vocabulario $/ \mathrm{CP}=$ Comprensión $/$ $\mathrm{SI}=$ Semejanzas $/ \mathrm{RP}=$ Adivinanzas $/ \mathrm{IN}$

$=$ Información $/ \mathrm{CD}=$ Claves $/ \mathrm{SB}=$ Búsqueda de símbolos $/ \mathrm{CN}=$ Animales $/ \mathrm{RM}=$ Matrices $/ \mathrm{COF}=$ Conceptos $/ \mathrm{CU}=$

Construcción por cubos $/ \mathrm{CF}=$ Completamiento de figuras / DI = Retención de Dígitos / $\mathrm{SQ}=$ Letras y números $/ \mathrm{AT}=$ Aritmética.

De acuerdo con la Figura 1, se puede observar que los coeficientes de regresión confirmaron los cuatro factores propuestos (ICV, IVP, IOP e IMO) y que todos presentan cargas factoriales muy altas. El Razonamiento Perceptivo presentó la mayor carga $(\#=0.98)$, mientras que la Comprensión Verbal y la Memoria Operativa tuvieron la misma carga entre ellas y muy próxima del IOP $(\#=0.97)$. Por último, la Velocidad de Procesamiento fue el factor que presentó la menor carga $(\#=0.83)$. Se destaca que además de los coeficientes haber sido muy altos, las cargas factoriales de los 15 subtest se ajustaron a la estructura de las puntuaciones compuestas presentadas por el WISC-IV. En 
ese sentido, las cargas de los subtest del ICV variaron entre 0.82 y 0.93 ; del IVP, entre 0.77 y 0.82 ; los subtest del IOP entre 0.7 y 0.84 y los subtest del IMO variaron entre 0.76 y 0.92 . A pesar de eso, es importante resaltar que las relaciones especificadas por el modelo tuvieron un peso significativo $(\mathrm{p}<0.001)$ sobre el factor en cuestión.

En seguida, fue investigada la posibilidad de que los subtests del WISC-IV evaluasen un factor general (factor g). Los resultados se presentan en la Figura 2.

\section{Figura 2}

Estructura factorial de la adaptación brasileña de WISC-IV considerando un factor general.

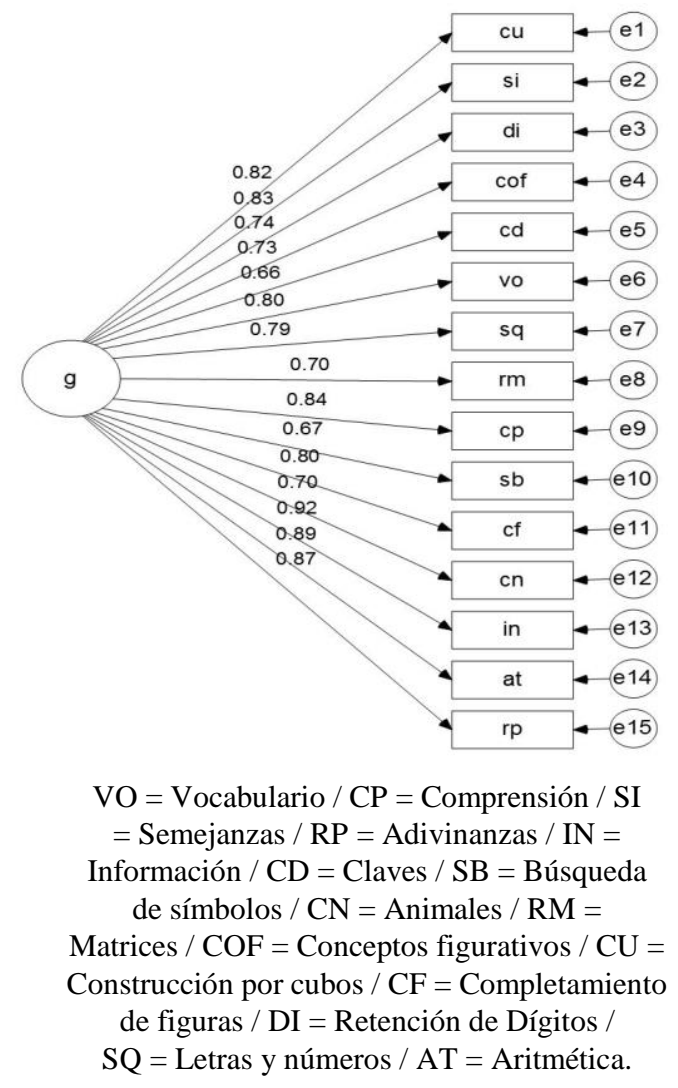

Considerando la existencia de un factor general, todos los subtest del WISC-IV presentaron cargas factoriales elevadas, variando de 0.66 a 0.92 . Pese a eso, hay que resaltar que los índices de bondad de ajuste no fueron satisfactorios, como se verá más adelante.

Finalmente, fue estudiado un modelo propuesto por Keith et al. (2006) con base en la teoría CHC. Investigando ese modelo, se pudo observar que la solución fue satisfactoria. Pese a eso, es importante destacar que fueron identificadas algunas cargas factoriales muy bajas y negativas, de modo que los coeficientes beta ajustados indicaron que el subtest Matrices presentó cargas positivas en Gf y negativas y bajas en $\mathrm{Gv}$, lo que contradice el modelo propuesto por los autores.

Por eso, posteriormente fue investigado el modelo de Keith et al. (2006), considerando el subtest $\mathrm{rm}$ de forma separada en los factores latentes Gv y Gf, y se observó que los valores presentaron un ajuste más satisfactorio al modelo, considerando rm en el factor latente Gv, a pesar de que las cargas factoriales hayan sido menores que en los modelos anteriormente estudiados y que todavía exista una con carga muy baja (subtest Buscar símbolos en el factor latente Gv). Los datos de ese modelo, considerando rm en el factor $\mathrm{Gv}$, se presentan en la Figura 3. 
Fabián Javier Marín Rueda, Acácia Aparecida Angeli dos Santos, Ana Paula Porto Noronha.

\section{Figura 3}

Estructura factorial de la adaptación brasileña de WISC-IV de acuerdo con un modelo CHC.

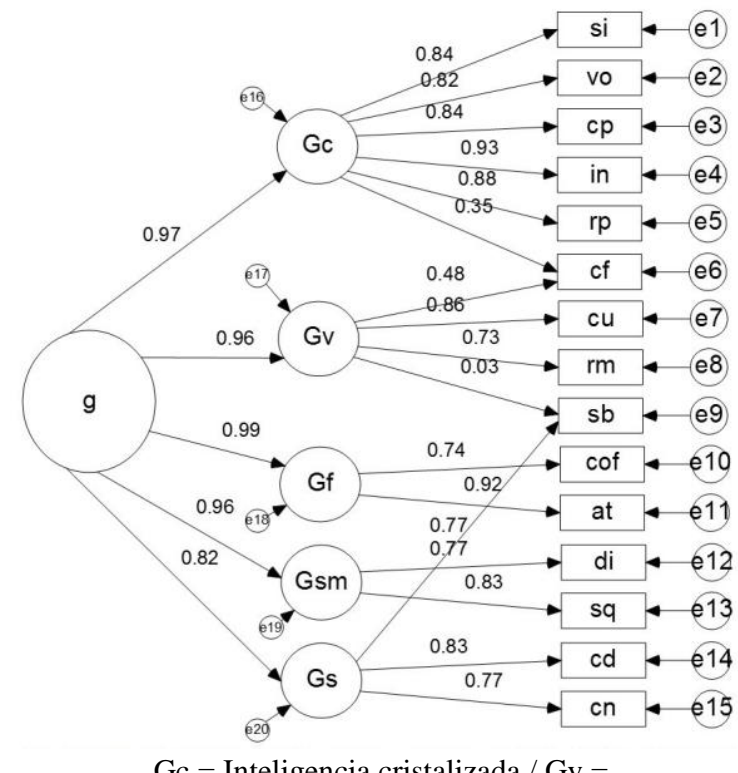

$\mathrm{Gc}=$ Inteligencia cristalizada $/ \mathrm{Gv}=$

Procesamiento visual / Gf = Inteligencia fluída /

Gsm = Memoria de corto plazo $/$ Gs = Rápidez

de procesamiento cognitivo / $\mathrm{VO}=$ Vocabulario /

$\mathrm{CP}=$ Comprensión $/ \mathrm{SI}=$ Semejanzas $/ \mathrm{RP}=$

Adivinanzas / IN = Información / CD = Claves /

$\mathrm{SB}=$ Búsqueda de símbolos $/ \mathrm{CN}=$ Animales $/$

$\mathrm{RM}=$ Matrices $/ \mathrm{COF}=$ Conceptos $/ \mathrm{CU}=$

Construcción por cubos $/ \mathrm{CF}=$ Completamiento

de figuras / DI = Retención de Dígitos /

$\mathrm{SQ}=$ Letras y números $/ \mathrm{AT}=$ Aritmética.

En la Figura 3, se observa que los coeficientes de regresión estandarizados confirman los cinco factores propuestos por Keith et al. (2006) con base en la teoría CHC (Inteligencia CristalizadaGc, Procesamiento Visual-Gv, Inteligencia Fluida-Gf, Memoria a Corto Plazo-Gsm y RApidez de Procesamiento Cognitivo-Gs) y que todos ellos también presentan cargas factoriales muy altas. La Gf presentó una carga de 0.99, lo que indica que el factor general podría ser explicado casi exclusivamente por el factor latente relacionado a la inteligencia fluida, lo que está de acuerdo con la teoría CHC. A su vez, el Gc presentó la segunda mayor carga $(\#=0.97)$ y Gv y Gsm presentaron cargas iguales $(\#=$ 0.96). Finalmente, Gs presentó la menor carga (\# = 0.82), pero también puede ser considerada bastante alta. En la Tabla 2 se presentan los índices de ajuste de los modelos considerados anteriormente.

Tabla 2

Índices de bondad de ajuste para los tres modelos

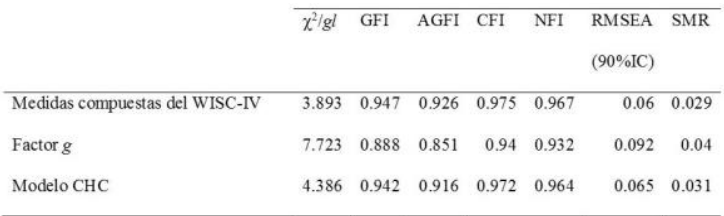

Fuente: elaboración propia

La Tabla 2 muestra que en el caso del modelo de factor general, en el cual se observaron cargas factoriales elevadas para los subtest, los índices calculados indican la falta de ajuste del modelo. Además, los índices CFI y NFI presentaron valores apenas aceptables y el valor de $\mathrm{c} 2 / \mathrm{gl}$ fue bastante mayor de lo admisible.

\section{Discusión}

El presente estudio tuvo como objetivo investigar la estructura del WISC-IV por medio del Análisis Factorial Confirmatorio, a fin de verificar la adecuación de algunos modelos teóricos entre los cuales se tomó en cuenta además del usado como base para su construcción, otro considerando el modelo CHC propuesto por Keith et al. (2006) y un tercero teniendo en cuenta la existencia de un factor general. En lo referente al concepto de inteligencia del WISC-IV, conviene resaltar que es definido como una capacidad global de pensar racionalmente, actuar de acuerdo con objetivos y relacionarse eficazmente con el medio. De esa forma, Wechsler (1949) definió la inteligencia no como una capacidad específica, sino global.

En relación con los resultados de este estudio, el ACF reveló que de los tres modelos investigados, el que mejor se ajustó fue el propuesto por las cuatro dimensiones del WISCIV, toda vez que los valores encontrados fueron muy altos, así como las cargas factoriales de los 15 subtest se ajustaron adecuadamente a la estructura de las puntuaciones. Con base en esto, es posible interpretar que la estructura 
propuesta por Wechsler (2003) fue adecuada para la muestra brasileña.

El segundo análisis consistió en el estudio de la posibilidad del WISC-IV de evaluar un factor general. Aunque los subtest hayan presentado cargas factoriales elevadas, variando de 0.66 a 0.92 , hay que resaltar que los índices de bondad de ajuste no fueron satisfactorios y el modelo fue rechazado categóricamente.

A su vez, se destaca que los índices de bondad de ajuste del modelo de puntuaciones compuestas del WISC-IV y del modelo CHC de Keith et al. (2006) se mostraron bastante semejantes, lo que no ocurrió en el análisis de Brenlla (2013). Sin embargo, todos los valores del modelo del WISC-IV presentaron resultados más favorables. También es importante recordar que el modelo de Keith et al. (2006) fue alterado, una vez que el subtest Matrices fue asociado al factor latente Gv. Además, las cargas factoriales de ese modelo fueron bastante inferiores al modelo de puntuaciones compuestas del WISCIV.

La relevancia de estudios de esta naturaleza se justifica por el hecho de que todos los instrumentos psicológicos deben ser analizados para determinar si efectivamente miden lo que se supone que miden. Es decir, el modelo que sirvió de base no fue necesariamente formalizado en una teoría, aunque Wechsler (1949) haya reconocido su simpatía por las afirmaciones de Spearman (1927). Los estudios brasileños con la 4.a edición de la Escala Wechsler son recientes y deben ser objeto de otras investigaciones.

Vale resaltar que aunque este estudio haya sido realizado con una muestra con distribución equilibrada en términos de edad y de sexo, se debe señalar como limitación el hecho de que la muestra no fue seleccionada al azar, toda vez que fue exigido que los padres autorizasen la participación de sus hijos y que los niños y adolescentes también estuviesen de acuerdo en participar de la investigación. Una agenda de estudios futuros con el WISC-IV deberá incluir evidencias de validez de criterio con grupos de niños con desarrollo atípico (trastorno de espectro autista, síndrome de Down, etc.) y con medidas de desempeño escolar. Finalmente, es importante considerar la posibilidad de realizar estudios sobre la estructura del WISC-IV en otros países, ya que es una de las medidas más utilizadas en el mundo para evaluación de la inteligencia.

\section{Referencias}

Arbuckle, J. L. (2009). Amos 18 User's Guide . Chicago, IL: SPSS Inc.

Arias, R. M. (1996). Psicometria: teoria de los tests psicologicos y educacionais . Madrid: Síntesis Psicología.

Bentler, P. M., \& Bonett, D. G. (1980). Significance tests and goodness-of-fit in the analysis of covariance structures. Psychological Bulletin, 88 (3), 588-606. http:// dx.doi.org/10.1037/0033-2909.88.3.588

Brenlla, M. E. (2013). Interpretación del WISC-IV. Puntuaciones compuestas y modelos CHC. Ciencias Psicológicas, 7 (2), 183-197.

Byrne, B. (2001). Structural equation modeling with AMOS: Basic concepts, applications, and programming . Nueva York: Psyclology Press, Taylor \& Francis Group.

Carroll, J. B. (1993). Human cognitive abilities: A survey of factor-analytic studies. Cambridge: Cambridge University Press.

Carroll, J. B. (1997). The three-stratum theory of cognitive abilities. En D. P. Flanagan, J. L. Genshaft \& P. L. Harrison (Eds.), Contemporary intellectual assessment: Theories, tests, and issues (pp. 122-130). Nueva York: Guilford Press.

Cattell, R. B. (1941). Some theoretical issues in adult intelligence testing. Psychological Bulletin, 38, 592.

Horn, J. L. (1985). Remodeling old models of intelligence. En B. B. Wolman (Ed.), Handbook of intelligence: Theories, measurement, and applications (pp. 267-300). Nueva York: Wiley.

Horn, J. L. (1991). Measurement of intellectual capabilities: A review of theory. En K. S. McGrew, J. K. Werder \& R. 
Fabián Javier Marín Rueda, Acácia Aparecida Angeli dos Santos, Ana Paula Porto Noronha.

W. Woodcock (Eds.), Woodcock-Johnson technical manual (pp. 197-232). Itasca, IL: Riverside.

Jensen, A. R. (1998). The g factor: The science of mental ability. Westport, CT: Praeger Publishers.

Keith, T. Z., Fine, J. G., Taub, G., Reynolds, M. R., \& Kranzler, J. H. (2006). Higher order, multisample, confirmatory factor analysis of the Weschler Intelligence Scale for Children-Fourth Edition: What does it measure? School Psychology Review, 35 (1), 108-127.

Schneider, W. J., \& McGrew, K. S. (2012). The Cattell-Horn-Carroll model of intelligence. En D. P. Flanagan \& P. L. Harrison (Eds.), Contemporary intellectual assessment: Theories, tests, and issues (pp. 99-144). Nueva York: Guilford.

Schweizer, K. (2010). Some guidelines concerning the modeling of traits and abilities in test construction. European Journal of Psychological Assessment, 26 (1), 1-2.

Spearman, C. (1904). "General Intelligence", objectively determined and measured. American Journal of Psychology , 15, 201-293.

Spearman, C. (1927). Las habilidades del hombre: su naturaleza y medición. Buenos Aires: Paidós.

Tabachnick, B. G., \& Fidell, L. S. (2001). Using multivariate statistics. Needham Heights, MA: Allyn \& Bacon.

Watkins, M. W., Canivez, G. L., James, T., James, K., \& Good, R. (2013). Construct validity of the WISC-IVUK with a large referred Irish sample. International Journal of School and Educational Psychology, 1 (2), 102-111.

Wechsler, D. (1949). Wechsler Intelligence Scale for Children. Nueva York: The Psychological Corporation.

Wechsler, D. (1974). Manual for the Wechsler Intelligence Scale for Children-Revised . Nueva York: Psychological Corporation.
Wechsler, D. (1991). Wechsler Intelligence Scale for Children (3. ${ }^{\circ}$ ed.). San Antonio, TX: The Psychological Corporation.

Wechsler, D. (2003). Wechsler Intelligence Scale for Children (4. ${ }^{\circ}$ ed.). San Antonio, TX: The Psychological Corporation.

Woodcock, R. R., McGrew, K. S., \& Schenk, F. A. (2007). Woodcock-Johnson III normative update technical manual . Itasca: Riverside.

\section{Notas}

* Artículo de investigación 